Psychological Medicine

cambridge.org/psm

\section{Correspondence}

Cite this article: García-Fernández L, RomeroFerreiro V, López-Roldán PD, Padilla S, CaleroSierra I, Monzó-García M, Pérez-Martín J, Rodriguez-Jimenez R (2022). Mental health impact of COVID-19 pandemic on Spanish healthcare workers. Psychological Medicine 52, 195-197. https://doi.org/10.1017/ S0033291720002019

Received: 15 May 2020

Revised: 18 May 2020

Accepted: 22 May 2020

First published online: 27 May 2020

Key words:

Acute stress; anxiety; COVID-19; depression; healthcare workers

Author for correspondence:

Lorena García-Fernández,

E-mail: lorena.garciaf@umh.es
(C) The Author(s), 2020. Published by Cambridge University Press. This is an Open Access article, distributed under the terms of the Creative Commons AttributionNonCommercial-NoDerivatives licence (http:// creativecommons.org/licenses/by-nc-nd/4.0/), which permits non-commercial re-use, distribution, and reproduction in any medium, provided the original work is unaltered and is properly cited. The written permission of Cambridge University Press must be obtained for commercial re-use or in order to create a derivative work.

\section{CAMBRIDGE UNIVERSITY PRESS}

\title{
Mental health impact of COVID-19 pandemic on Spanish healthcare workers
}

\author{
Lorena García-Fernández ${ }^{1,2,3}$ (D), Verónica Romero-Ferreiro ${ }^{3,4,5}$, \\ Pedro David López-Roldán², Sergio Padilla ${ }^{1,6}$, Irene Calero-Sierra², \\ María Monzó-García², Jorge Pérez-Martín² and Roberto Rodriguez-Jimenez 3,5,7
}

${ }^{1}$ Clinical Medicine Department, Universidad Miguel Hernández, Ctra, de Valencia, Km 87, 03550 San Juan, Alicante, Spain; ${ }^{2}$ Department of Psychiatry, Hospital Universitario de San Juan, Ctra, N-332, s/n, 03550 San Juan, Alicante, Spain; ${ }^{3}$ CIBERSAM (Biomedical Research Networking Centre in Mental Health), Madrid, Spain; ${ }^{4}$ Brain Mapping Unit, Instituto Pluridisciplinar, Universidad Complutense de Madrid (UCM), Paseo Juan XXIII, 1, 28040 Madrid, Spain; ${ }^{5}$ Department of Psychiatry, Instituto de Investigación Sanitaria Hospital 12 de Octubre (imas 12), Av. Córdoba s/n, 28041, Madrid, Spain; ${ }^{6}$ Infectious Diseases Unit, Hospital Universitario de Elche, Carrer Almazara, 11, 03203 Elche, Alicante, Spain and ${ }^{7}$ Facultad de Medicina, Universidad Complutense de Madrid (UCM), Plaza Ramón y Cajal, s/n, 28040 Madrid, Spain

Spain has the highest percentage of healthcare workers (HCW) infected with SARS-CoV-2 (WHO, 2019). This has led to significant concern among HCW precipitating emotional responses of anxiety, depression, and acute stress. We aimed to (1) explore differential presence of these symptoms among HCW compared with non-HCW; (2) compare their presence in the different health system roles; and (3) study the relationship between the emotional state of HCW and environmental variables.

Participants conducted a national self-reported online questionnaire starting on 29 March to 5 April 2020, which covers the peak of the infection (WHO, 2019), the questionnaire was distributed by social networks, applying an exponential non-discriminative snowball sampling (Liu et al., 2020; Roy et al., 2020; Wang et al., 2020).

HCW were eligible if: (a) they worked in a hospital or outpatient clinic, (b) had been occupationally active since the debut of the first case in Spain and (c) were aged between 18 and 65 . We categorized the final 781 participants into: 385 physicians (169 trainees and 215 seniors), 233 nurses, and 164 other professionals. Participants (1006) were allocated in the non-HCW if: (a) had been occupationally active since the debut of the first case in Spain and (b) were between 18 and 65 years old. The presence of a current or past mental disorder reported was considered exclusion criteria in both samples. Informed consent was provided. The study was approved by the ethics committee.

Sociodemographic information, as well as whether responders presented symptoms compatible with COVID-19 (suspected cases) or had undergone PCR with a positive result (confirmed cases) was required. Moreover, perception of the quality of the information received (insufficient/adequate/excessive) as well as effectiveness of the protection measures provided (insufficient/adequate/excessive) were included. The questionnaire included three scales to assess anxiety, depression, and acute stress: Hamilton Anxiety Scale (HARS) (Hamilton, 1959), Beck Depression Inventory (BDI) (Bech, 1988), and the Acute Stress Disorder Inventory (ASDI): consisting of a list of symptoms based on the clinical criteria of Acute Stress Disorder in the DSM-5 (APA, 2013).

\section{Anxiety, depression, and acute stress in the study groups}

Regarding anxiety symptoms $(F(1,1783)=0.93, p=0.34)$, the HCW group (M 18.2, s.D. 10.4) did not show significant higher symptoms of anxiety than non-HCW (M 16.9, s.D. 10.3). In depression, results showed no differences in BDI scores $(F(1,1780)=0.16, p=0.68)$ in HCW (M 4.0, s.D. 3.8) compared to non-HCW (M 3.6, s.D. 3.9). However, when clinical cut-off score of 4 (absent or minimal depression $v$. mild/moderate/severe depression) are applied to BDI responses, a trend toward greater depressive symptoms in HCW is observed $\left(\chi^{2}=2.9\right.$, $p=0.09)$.

Finally, HCW showed higher symptoms of acute stress $(F(1,1745)=8.1, p=0.004)$ with higher ASDI scores (M 4.9, s.D. 3.1) than non-HCW (M 4.3, s.D. 3.1).

\section{Comparisons according to the role within the healthcare system}

Nurses scored higher in all emotional assessments [anxiety: $21.3(10.9) v .16 .6$ (9.6) v. 17.3 (10.4), $p<0.001$; depression 4.5 (4.2) v. 3.2 (3.1) v. 3.4 (3.4), $p<0.03$; acute stress 5.5(3.2) v. 4.8 (3.0) v. 4.4 (3.3), $p<0.009$ ] than physicians and other professionals, respectively. No 
Table 1. Relation between anxiety, depression, and acute stress symptoms and COVID-19, level of information and level of protection

\begin{tabular}{|c|c|c|c|c|c|c|c|c|c|c|c|c|}
\hline & \multicolumn{4}{|c|}{ Anxiety (HARS score) ${ }^{a}$} & \multicolumn{4}{|c|}{ Depression (BDI score) ${ }^{\mathrm{b}}$} & \multicolumn{4}{|c|}{ Acute stress (ASDI score) ${ }^{c}$} \\
\hline & Mean & \multicolumn{2}{|c|}{ Difference (Cl 95\%) } & $p$ & Mean & \multicolumn{2}{|c|}{ Difference (Cl 95\%) } & $p$ & Mean & \multicolumn{2}{|c|}{ Difference (Cl 95\%) } & $p$ \\
\hline Absent & 17.51 & Ref & - & - & 4.31 & Ref & - & - & 4.58 & Ref & - & - \\
\hline Symptomatic suspected & 21.01 & +3.39 & $(1.84-4.94)$ & 0.001 & 5.20 & +0.89 & $(0.25-1.54)$ & 0.003 & 5.45 & +0.83 & $(0.36-1.30)$ & 0.001 \\
\hline Microbiologically confirmed & 27.59 & +11.14 & $(7.01-15.26)$ & 0.001 & 8.20 & +3.71 & $(1.91-5.25)$ & 0.001 & 6.56 & +1.57 & $(0.23-2.90)$ & 0.015 \\
\hline \multicolumn{13}{|l|}{ COVID-19 information } \\
\hline Excessive & 16.73 & Ref & - & - & 4.34 & +0.42 & $(-0.15$ to 0.98$)$ & 0.234 & 4.61 & +0.38 & $(-0.03$ to 0.78$)$ & 0.170 \\
\hline Adequate & 16.63 & +0.20 & $(-2.35$ to 2.75$)$ & 1.000 & 3.85 & Ref & - & - & 4.21 & Ref & - & - \\
\hline Insufficient & 20.53 & +3.49 & $(0.94-6.03)$ & 0.003 & 5.14 & +1.20 & $(0.69-1.72)$ & 0.001 & 5.27 & +1.02 & $(0.64-1.39)$ & 0.001 \\
\hline \multicolumn{13}{|l|}{ COVID-19 protection } \\
\hline Excessive & 20.63 & Ref & - & - & 6.55 & +2.29 & $(0.17-4.42)$ & 0.029 & 5.90 & +1.46 & $(-0.06$ to 2.99$)$ & 0.065 \\
\hline Adequate & 15.82 & -3.70 & $(-8.78$ to -1.38$)$ & 0.243 & 3.83 & Ref & - & - & 4.13 & Ref & - & - \\
\hline Insufficient & 18.81 & +2.65 & (1.44 to -3.85$)$ & 0.001 & 4.66 & +0.80 & $(0.30-1.31)$ & 0.001 & 4.90 & +0.66 & (0.29 to -1.02$)$ & 0.001 \\
\hline
\end{tabular}

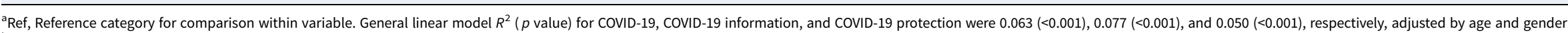

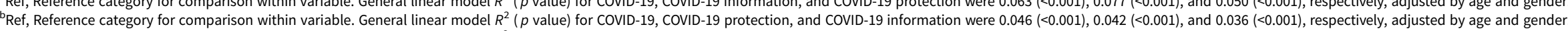

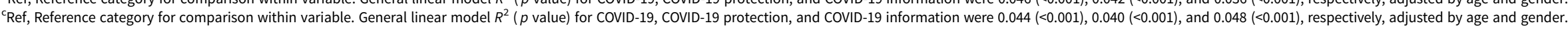


significant differences were found between physicians and other health professionals in all three clinical symptoms.

According to the degree of expertise within physicians, when clinical cut-offs score of 4 are applied to BDI, significant differences were found with up to $40.8 \%$ of trainees fulfilling scores for depression compared to $30.7 \%$ of specialists $(p=0.04)$. No differences in acute stress symptoms or anxiety were found between levels of expertise.

\section{Relation between emotional state and COVID-19, level of information, and level of protection}

Mean and CI 95\% are presented in Table 1. A confirmatory diagnosis of the disease increased the average HARS score $(p<0.001)$, BDI score $(p=0.001)$, and ASDI score $(p=0.015)$, compared with the presence of suspected disease. This latter increased the HARS $(p=0.001)$, BDI $(p=0.003)$, and ASDI $(p=0.001)$ means compared to healthy HCW. Regarding the information received, those participants considering they were provided insufficient information showed higher HARS $(p=0.003)$, BDI $(p=$ $0.001)$, and ASDI $(p=0.001)$ scores than those respondents who consider it adequate. No differences were found between adequate and excessive information in any of the measures. According to the protection measures, participants who considered the protection insufficient showed an increased average of HARS $(p=0.001)$, BDI $(p=0.001)$, and ASDI $(p=0.001)$ scores than those who consider it adequate. Finally, the excesses of protection perceived increased BDI scores $(p=0.029)$ than those who considered it adequate. No differences on HARS or ASDI scores between adequate and excessive protection were found.

Subsequent analyses of variance are corrected for age and gender.

Findings suggest that COVID-19 has greater impact on the mental health of HCW than in non-HCW. Nurses and physician trainees are the most vulnerable groups. Adequate information and availability of protective measures are associated with emotional wellbeing.
Acknowledgements. We thank all participants, especially frontline clinicians who have kindly responded to the survey.

Financial support. This research received no specific grant from any funding agency, commercial or not-for-profit sectors.

Conflicts of interest. Dr R. Rodriguez-Jimenez has been a consultant for, spoken in activities of, or received grants from: Instituto de Salud Carlos III, Fondo de Investigación Sanitaria (FIS), Centro de Investigación Biomédica en Red de Salud Mental (CIBERSAM), Madrid Regional Government (S2010/ BMD-2422 AGES; S2017/BMD-3740), JanssenCilag, Lundbeck, Otsuka, Pfizer, Ferrer, Juste, Takeda, Exeltis, Angelini, and Casen-Recordati. All other authors declare that they have no conflicts of interest.

\section{References}

American Psychiatric Association, \& American Psychiatric Association. (2013). Diagnostic and statistical manual of mental disorders: DSM-5. Arlington, VA: American Psychiatric Publication.

Bech, P. (1988). Rating scales for mood disorders: Applicability, consistency, and construct validity. Acta Psychiatrica Scandinavica, 78(S345), 45-55.

Hamilton, M. A. X. (1959). The assessment of anxiety states by rating. British Journal of Medical Psychology, 32(1), 50-55.

Liu, Q., Luo, D., Haase, J. E., Guo, Q., Wang, X. Q., Liu, S., .. Yang, B. X. (2020). The experiences of health-care providers during the COVID-19 crisis in China: A qualitative study. The Lancet Global Health, 8, e790-798.

Roy, D., Tripathy, S., Kar, S. K., Sharma, N., Verma, S. K., \& Kaushal, V. (2020). Study of knowledge, attitude, anxiety \& perceived mental healthcare need in Indian population during COVID-19 pandemic. Asian Journal of Psychiatry, 51, 102083.

Wang, C., Pan, R., Wan, X., Tan, Y., Xu, L., Ho, C. S., \& Ho, R. C. (2020). Immediate psychological responses and associated factors during the initial stage of the 2019 coronavirus disease (COVID-19) epidemic among the general population in China. International Journal of Environmental Research and Public Health, 17(5), 1729.

World Health Organization. (2019). Global influenza strategy 2019-2030. World Health Organization. https://apps.who.int/iris/handle/10665/ 311184. License: CC BY-NC-SA 3.0 IGO. 